\title{
Radiative decays of the $\mathbf{Y}(1 S)$ to a pair of charged hadrons
}

S. B. Athar, ${ }^{1}$ P. Avery, ${ }^{1}$ L. Breva-Newell,,${ }^{1}$ R. Patel, ${ }^{1}$ V. Potlia, ${ }^{1}$ H. Stoeck, ${ }^{1}$ J. Yelton,,${ }^{1}$ P. Rubin, ${ }^{2}$ C. Cawlfield, ${ }^{3}$ B. I. Eisenstein, ${ }^{3}$ G. D. Gollin, ${ }^{3}$ I. Karliner, ${ }^{3}$ D. Kim,${ }^{3}$ N. Lowrey, ${ }^{3}$ P. Naik, ${ }^{3}$ C. Sedlack, ${ }^{3}$ M. Selen, ${ }^{3}$ E. J. White, ${ }^{3}$ J. Williams, ${ }^{3}$ J. Wiss, ${ }^{3}$ D. M. Asner, ${ }^{4,5}$ K. W. Edwards, ${ }^{4,5}$ D. Besson, ${ }^{6}$ T. K. Pedlar, ${ }^{7}$ D. Cronin-Hennessy, ${ }^{8}$ K. Y. Gao, ${ }^{8}$ D. T. Gong, ${ }^{8}$ J. Hietala, ${ }^{8}$ Y. Kubota, ${ }^{8}$ T. Klein, ${ }^{8}$ B. W. Lang, ${ }^{8}$ S. Z. Li, ${ }^{8}$ R. Poling, ${ }^{8}$ A. W. Scott,${ }^{8}$ A. Smith, ${ }^{8}$ S. Dobbs, ${ }^{9}$ Z. Metreveli, ${ }^{9}$ K. K. Seth, ${ }^{9}$ A. Tomaradze, ${ }^{9}$ P. Zweber, ${ }^{9}$ J. Ernst, ${ }^{10}$ K. Arms,${ }^{11}$ H. Severini, ${ }^{12}$ S. A. Dytman, ${ }^{13}$ W. Love,${ }^{13}$ S. Mehrabyan, ${ }^{13}$ J. A. Mueller, ${ }^{13}$ V. Savinov, ${ }^{13} \mathrm{Z}$. Li, ${ }^{14}$ A. Lopez, ${ }^{14}$ H. Mendez ${ }^{14}$ J. Ramirez, ${ }^{14}$ G. S. Huang, ${ }^{15}$ D. H. Miller, ${ }^{15}$ V. Pavlunin, ${ }^{15}$ B. Sanghi, ${ }^{15}$ I. P. J. Shipsey, ${ }^{15}$ G. S. Adams, ${ }^{16}$ M. Cravey, ${ }^{16}$ J. P. Cummings, ${ }^{16}$ I. Danko, ${ }^{16}$ J. Napolitano, ${ }^{16}$ Q. He, ${ }^{17}$ H. Muramatsu, ${ }^{17}$ C.S. Park,,${ }^{17}$ E. H. Thorndike, ${ }^{17}$ T. E. Coan, ${ }^{18}$ Y. S. Gao, ${ }^{18}$ F. Liu, ${ }^{18}$ R. Stroynowski, ${ }^{18}$ M. Artuso, ${ }^{19}$ C. Boulahouache, ${ }^{19}$ S. Blusk, ${ }^{19}$ J. Butt, ${ }^{19}$ O. Dorjkhaidav, ${ }^{19} \mathrm{~J} . \mathrm{Li},{ }^{19} \mathrm{~N}$. Menaa, ${ }^{19}$ R. Mountain, ${ }^{19}$ R. Nandakumar, ${ }^{19}$ K. Randrianarivony, ${ }^{19}$ R. Redjimi,${ }^{19}$ R. Sia, ${ }^{19}$ T. Skwarnicki, ${ }^{19}$ S. Stone, ${ }^{19}$ J. C. Wang, ${ }^{19}$ K. Zhang, ${ }^{19}$ S. E. Csorna, ${ }^{20}$ G. Bonvicini, ${ }^{21}$ D. Cinabro, ${ }^{21}$ M. Dubrovin, ${ }^{21}$ A. Bornheim, ${ }^{22}$ S. P. Pappas, ${ }^{22}$ A. J. Weinstein,${ }^{22}$ R. A. Briere, ${ }^{23}$ G. P. Chen, ${ }^{23}$ J. Chen, ${ }^{23}$ T. Ferguson, ${ }^{23}$ G. Tatishvili, ${ }^{23}$ H. Vogel, ${ }^{23}$ M. E. Watkins, ${ }^{23}$ J. L. Rosner, ${ }^{24}$ N.E. Adam, ${ }^{25}$ J.P. Alexander, ${ }^{25}$ K. Berkelman,${ }^{25}$ D. G. Cassel, ${ }^{25}$ V. Crede,${ }^{25}$ J. E. Duboscq, ${ }^{25}$ K. M. Ecklund, ${ }^{25}$ R. Ehrlich ${ }^{25}$ L. Fields, ${ }^{25}$ R. S. Galik, ${ }^{25}$ L. Gibbons, ${ }^{25}$ B. Gittelman, ${ }^{25}$ R. Gray, ${ }^{25}$ S. W. Gray, ${ }^{25}$ D. L. Hartill, ${ }^{25}$ B. K. Heltsley, ${ }^{25}$ D. Hertz, ${ }^{25}$ C. D. Jones,${ }^{25}$ J. Kandaswamy, ${ }^{25}$ D. L. Kreinick,${ }^{25}$ V. E. Kuznetsov, ${ }^{25}$ H. Mahlke-Krüger ${ }^{25}$ T. O. Meyer ${ }^{25}$ P. U. E. Onyisi, ${ }^{25}$ J. R. Patterson, ${ }^{25}$ D. Peterson ${ }^{25}$ E. A. Phillips ${ }^{25}$ J. Pivarski, ${ }^{25}$ D. Riley,${ }^{25}$ A. Ryd ${ }^{25}$ A. J. Sadoff, ${ }^{25}$ H. Schwarthoff, ${ }^{25}$ X. Shi, ${ }^{25}$ M. R. Shepherd, ${ }^{25}$ S. Stroiney, ${ }^{25}$ W. M. Sun,${ }^{25}$ D. Urner, ${ }^{25}$ T. Wilksen, ${ }^{25}$ K. M. Weaver, ${ }^{25}$ and M. Weinberger ${ }^{25}$

\section{(CLEO Collaboration)}

\author{
${ }^{1}$ University of Florida, Gainesville, Florida 32611, USA \\ ${ }^{2}$ George Mason University, Fairfax, Virginia 22030, USA \\ ${ }^{3}$ University of Illinois, Urbana-Champaign, Illinois 61801, USA \\ ${ }^{4}$ Carleton University, Ottawa, Ontario, Canada K1S 5B6 \\ ${ }^{5}$ The Institute of Particle Physics, Canada \\ ${ }^{6}$ University of Kansas, Lawrence, Kansas 66045, USA \\ ${ }^{7}$ Luther College, Decorah, Iowa 52101, USA \\ ${ }^{8}$ University of Minnesota, Minneapolis, Minnesota 55455, USA \\ ${ }^{9}$ Northwestern University, Evanston, Illinois 60208, USA \\ ${ }^{10}$ State University of New York at Albany, Albany, New York 12222, USA \\ ${ }^{11}$ Ohio State University, Columbus, Ohio 43210, USA \\ ${ }^{12}$ University of Oklahoma, Norman, Oklahoma 73019, USA \\ ${ }^{13}$ University of Pittsburgh, Pittsburgh, Pennsylvania 15260, USA \\ ${ }^{14}$ University of Puerto Rico, Mayaguez, Puerto Rico 00681 \\ ${ }^{15}$ Purdue University, West Lafayette, Indiana 47907, USA \\ ${ }^{16}$ Rensselaer Polytechnic Institute, Troy, New York 12180, USA \\ ${ }^{17}$ University of Rochester, Rochester, New York 14627, USA \\ ${ }^{18}$ Southern Methodist University, Dallas, Texas 75275, USA \\ ${ }^{19}$ Syracuse University, Syracuse, New York 13244, USA \\ ${ }^{20}$ Vanderbilt University, Nashville, Tennessee 37235, USA \\ ${ }^{21}$ Wayne State University, Detroit, Michigan 48202, USA \\ ${ }^{22}$ California Institute of Technology, Pasadena, California 91125, USA \\ ${ }^{23}$ Carnegie Mellon University, Pittsburgh, Pennsylvania 15213, USA \\ ${ }^{24}$ Enrico Fermi Institute, University of Chicago, Chicago, Illinois 60637, USA \\ ${ }^{25}$ Cornell University, Ithaca, New York 14853, USA \\ (Received 4 October 2005; published 3 February 2006)
}

\begin{abstract}
Using data obtained with the CLEO III detector, running at the Cornell Electron Storage Ring (CESR), we report on a new study of exclusive radiative $Y(1 \mathrm{~S})$ decays into the final states $\gamma \pi^{+} \pi^{-}, \gamma K^{+} K^{-}$, and $\gamma p \bar{p}$. We present branching ratio measurements for the decay modes $\mathrm{Y}(1 \mathrm{~S}) \rightarrow \gamma f_{2}(1270), \mathrm{Y}(1 \mathrm{~S}) \rightarrow$ $\gamma f_{2}^{\prime}(1525)$, and $\mathrm{Y}(1 \mathrm{~S}) \rightarrow \gamma K^{+} K^{-}$; helicity production ratios for $f_{2}(1270)$ and $f_{2}^{\prime}(1525)$; upper limits for the decay $Y(1 \mathrm{~S}) \rightarrow \gamma f_{J}(2200)$, with $f_{J}(2220) \rightarrow \pi^{+} \pi^{-}, K^{+} K^{-}, p \bar{p}$; and an upper limit for the decay $\Upsilon(1 \mathrm{~S}) \rightarrow \gamma X(1860)$, with $X(1860) \rightarrow \gamma p \bar{p}$.
\end{abstract}




\section{INTRODUCTION}

Radiative decays of heavy quarkonia, where a photon replaces one of the three gluons from the strong decay of, for example, the $J / \psi$ or $Y(1 S)$, are useful in studying color-singlet two-gluon systems. The two gluons can, among other things, hadronize into a meson, ${ }^{1}$ or directly form a glueball. ${ }^{2}$ Further information on radiative decays of heavy quarkonia can be found in [44].

Light-meson production in $J / \psi$ two-body radiative decays has been experimentally well established with branching fractions at the $10^{-3}$ level, based largely on evidence provided by radiative decays to a pair of hadrons. ${ }^{3}$ The production ratios of the available helicity states have been measured for the tensor mesons $f_{2}(1270)$ [46-49] and $f_{2}^{\prime}(1525)[50,51]$ in $J / \psi$ two-body radiative decays and agree with theoretical predictions [52,53]. In 1996, the BES Collaboration reported the observation of the $f_{J}(2220)$ in $J / \psi$ two-body radiative decays, and measured product branching fractions, $\mathcal{B}\left(J / \psi \rightarrow \gamma f_{J}(2220)\right) \times$ $\mathcal{B}\left(f_{J}(2220) \rightarrow h^{+} h^{-}\right) \quad$ (we use the convention $h=$ $\pi, K, p)$, of the order of $10^{-5}$ [54]. Much excitement was generated at the time because it is possible to interpret the $f_{J}(2220)$ as a glueball. A candidate similar to $f_{J}(2220)$ was reported in 1986 by the Mark-III Collaboration in the $K \bar{K}$ mode [55], but was not confirmed by the DM2 Collaboration [56]. Recently, BES reported the existence of a new particle, the $X(1860)$, observed by its decay $J / \psi \rightarrow \gamma X(1860) \rightarrow p \bar{p}$ [57], a result that is currently being interpreted [58-64].

The experimental observation of radiative $\mathrm{Y}(1 \mathrm{~S})$ decays is challenging because their rate is suppressed to a level of

$$
\left(\frac{q_{b}}{q_{c}}\right)^{2}\left(\frac{m_{c}}{m_{b}}\right)^{2} \approx 0.025
$$

of the corresponding rate of $J / \psi$ radiative decays. This factor arises because the quark-photon coupling is proportional to the electric charge, and the quark propagator is

\footnotetext{
${ }^{1}$ Several authors have studied meson production in $Y(1 S)$ radiative decays, giving predictions for branching and helicity production ratios. The heavy-quarkonium system is usually described by nonrelativistic QCD [1], while the gluonic hadronization has been treated using soft collinear effective theory [2], gluon distribution amplitudes [3], and perturbative QCD [4,5].

${ }^{2}$ Glueballs are a natural consequence of QCD, and predictions of their properties have been made using different approaches, such as potential models [6-8], lattice QCD calculations [9-12], bag models [13-16], flux-tube models [17], the QCD sum rules [18], the Bethe-Salpeter (B-S) equation [19,20], QCD factorization formalism models [21,22], weakly bound-state models [23], and a three-dimensional relativistic equation [24]. However, despite intense experimental searches [25-31], there is no conclusive experimental evidence of their direct observation, although there are strong indications that glueballs contribute to the rich light scalar [32-41] and tensor [42,43] spectrums.

${ }^{3}$ We refer to the Particle Data Group [45] for a summary of $J / \psi$ radiative decays.
}

roughly proportional to $1 / \mathrm{m}$ for low momentum quarks. Taking into account the total widths [45] of $J / \psi$ and $\Upsilon(1 \mathrm{~S})$, the branching fraction of a particular $\mathrm{Y}(1 \mathrm{~S})$ radiative decay mode is expected to be around 0.04 of the corresponding $J \psi$ branching fraction. In 1999, CLEO II made the first observation of a radiative $Y(1 S)$ decay to a pair of hadrons [65], which was consistent with $\mathrm{Y}(1 \mathrm{~S}) \rightarrow$ $\gamma f_{2}(1270)$, where $f_{2}(1270) \rightarrow \pi \pi$. Comparing the measured branching fraction to the $J / \psi \rightarrow \gamma f_{2}(1270)$ branching fraction, a suppression factor of $0.06 \pm 0.03$ was obtained. Recent theoretical works [2,3] predict a suppression factor between $0.06-0.18$ for this mode, and favor the production of $f_{2}(1270)$ in a helicity-0 state. After the BES result for the $f_{J}(2220)$ in radiative $J / \psi$ decays, a corresponding search was performed by CLEO II in the radiative $Y(1 S)$ system [66] and limits were put on some of the glueball candidates' product branching ratios.

In this paper, we use the CLEO III Y(1S) data sample, which has 15 times higher statistics and better particle identification than the CLEO II data sample, to probe the color-singlet two-gluon spectrum by measuring the system's invariant mass using its decays to $\pi^{+} \pi^{-}, K^{+} K^{-}$, and $p \bar{p}$. Further details of this analysis can be found elsewhere [67].

\section{CLEO III DETECTOR, DATA, AND MONTE CARLO SIMULATED SAMPLE}

The CLEO III detector is a versatile multipurpose particle detector described more fully in [68]. It is centered on the interaction region of Cornell Electron Storage Ring (CESR). From the $e^{+} e^{-}$interaction region radially outward it consists of a silicon strip vertex detector and a wire drift chamber used to measure the position, momenta, and specific ionization energy losses $(d E / d x)$ of charged tracks based on their fitted path in a $1.5 \mathrm{~T}$ solenoidal magnetic field and the amount of charge deposited on the drift chamber wires. The silicon vertex detector and drift chamber tracking system achieves a charged particle momentum resolution of $0.35 \%(1 \%)$ at $1 \mathrm{GeV} / \mathrm{c}(5 \mathrm{GeV} / \mathrm{c})$ and a $d E / d x$ resolution of $6 \%$. Beyond the drift chamber is a ring imaging Cherenkov detector, $\mathrm{RICH}$, which covers $80 \%$ of the solid angle and is used to further identify charged particles by giving for each mass hypothesis the likelihood of a fit to the Cherenkov radiation pattern. After the RICH is a crystal calorimeter (CC) that covers $93 \%$ of the solid angle. The $\mathrm{CC}$ has a resolution of $2.2 \%$ (1.5\%) for $1 \mathrm{GeV}(5 \mathrm{GeV})$ photons. After the $\mathrm{CC}$ is a superconducting solenoid coil that provides the magnetic field, followed by iron flux return plates with wire chambers interspersed in three layers at 3,5, and 7 hadronic interaction lengths to provide muon identification.

The data sample has an integrated luminosity of $1.13 \mathrm{fb}^{-1}$ taken at the $\mathrm{Y}(1 \mathrm{~S})$ energy, $\sqrt{s}=9.46 \mathrm{GeV}$, which corresponds to $21.2 \pm 0.2$ million $Y(1 \mathrm{~S})$ decays 
[69] and $3.49 \mathrm{fb}^{-1}$ taken at the $\mathrm{Y}(4 \mathrm{~S})$ energy, $\sqrt{s}=$ $10.56 \mathrm{GeV}$, used to model the underlying continuum present in the $\mathrm{Y}(1 \mathrm{~S})$ data sample. The continuum background modeling is important because continuum background processes such as $e^{+} e^{-} \rightarrow \gamma \rho$ with $\rho \rightarrow \pi^{+} \pi^{-}$, $e^{+} e^{-} \rightarrow \gamma \phi$ with $\phi \rightarrow K^{+} K^{-}$, and direct $e^{+} e^{-} \rightarrow$ $\gamma h^{+} h^{-}$have the same topology as the signal events we are investigating.

Efficiencies are evaluated using a Monte Carlo simulation of the process [70] and a GEANT-based [71] detector response. Monte Carlo samples of $e^{+} e^{-} \rightarrow \gamma X$ with $X \rightarrow$ $h^{+} h^{-}$are generated at both the $Y(1 \mathrm{~S})$ and $Y(4 \mathrm{~S})$ energies with uniform angular distributions and flat $h^{+} h^{-}$invariantmass distributions from threshold to $3.5 \mathrm{GeV} / \mathrm{c}^{2}$.

\section{EVENT SELECTION}

Events which satisfy the CLEO III trigger [72] are then required to meet the following analysis requirements: (a) There are exactly two charged tracks that trace back to the beam spot and have good quality track fits and $d E / d x$ information. (b) There is exactly one CC shower that is unmatched to any track and whose energy, $E_{\gamma}$, is greater than $4 \mathrm{GeV}$. The efficiency of these initial basic event requirements is approximately $65 \%$ for our signal events.

Each event is also required to be consistent with having the 4-momentum of the initial $e^{+} e^{-}$system by demanding that the chi squared from a kinematic fit to the following constraint,

$$
\vec{p}_{h^{+} h^{-}}+\left(2 E_{\text {beam }}-E_{h^{+} h^{-}}\right) \hat{p}_{\gamma}=\vec{p}_{C M},
$$

be less than 100, where $\vec{p}_{h^{+} h^{-}}$is the di-hadron momentum, $E_{h^{+} h^{-}}$is the di-hadron energy, $E_{\text {beam }}$ is the beam energy, $\hat{p}_{\gamma}$ is the photon's direction, and $\vec{p}_{C M}$ is the momentum of the $e^{+} e^{-}$system [which has a magnitude of a few $\mathrm{MeV} / \mathrm{c}$ because of the small $(\approx 2 \mathrm{mrad})$ crossing angle of the $e^{+}$and $e^{-}$beams]. This requirement is approximately $99 \%$ efficient, but is effective in eliminating almost all background of the wrong topology.

Equation (1) is a 3-constraint subset of the 4-momentum constraint and has the convenient property of avoiding the use of the measured photon energy, which has an asymmetric measurement uncertainty. We improve the measurement of the di-hadron 4-momenta (the di-hadron invariantmass resolution becomes $3.2,2.6$, and $2.0 \mathrm{MeV} / \mathrm{c}^{2}$ for the pion, kaon, and proton modes, respectively) by using the constraint in Eq. (1), and then demanding that

$$
0.950<\left(E_{h^{+} h^{-}}+E_{\gamma}\right) / 2 E_{\text {beam }}<1.025 .
$$

Strong electron and muon vetoes are imposed to suppress the abundant QED processes $e^{+} e^{-} \rightarrow \gamma e^{+} e^{-}$and $e^{+} e^{-} \rightarrow \gamma \mu^{+} \mu^{-}$. To reject $e^{+} e^{-} \rightarrow \gamma e^{+} e^{-}$, we require each track to have a matched CC shower with an energy $E$, together with a measured momentum $p$, such that $\mid E / p-$ $0.95 \mid>0.1$, and that the combined RICH and $d E / d x$ likelihood for $h$ be higher than the combined likelihood for $e$. To reject $e^{+} e^{-} \rightarrow \gamma \mu^{+} \mu^{-}$, we require that neither track produce a signal in the five hadronic interaction lengths of the muon system. For the $\pi^{+} \pi^{-}$mode, where muon background is a particular problem because of the similar pion and muon masses, we further require that both tracks must be within the barrel part of the muon chambers $(|\cos \theta|<0.7)$, and both have $p>1 \mathrm{GeV} / \mathrm{c}$. To increase the solid-angle acceptance of the detector and improve the overall muon suppression efficiency with virtually no increase in muon fakes, we flag an event as "not muonic" and remove the muon suppression requirements if either track deposits more than $600 \mathrm{MeV}$ in the $\mathrm{CC}$.

Events that satisfy all the above requirements are then identified as either $\pi^{+} \pi^{-}, K^{+} K^{-}$, or $p \bar{p}$ using the RICH and $d E / d x$ information. Since the ratios $\pi^{+} \pi^{-} / K^{+} K^{-}$ and $K^{+} K^{-} / p \bar{p}$ are much larger than 1 for these types of events, in the three cases in which we try to reduce the background from a lower-mass hadron, we also use the chisquared value from the kinematic constraint in Eq. (1) to identify the event type. Since the constraint involves the dihadron energy, the chi-squared value is sensitive to the hadronic masses. After these procedures, the particle identification efficiencies (fake rates) are $90 \%(0.31 \%), 99 \%$ $(0.03 \%), 98 \%(0.10 \%)$ for kaons (pions faking kaons), protons (pions faking protons), and protons (kaons faking protons), respectively.

\section{DETERMINATION OF SIGNALS AND THEIR SPIN ASSIGNMENTS}

The overall reconstruction efficiencies as determined by Monte Carlo simulations, including both event selection and analysis cuts, are $43 \%, 48 \%$, and $56 \%$ for the $\mathrm{Y}(1 \mathrm{~S})$ radiative decays to $\pi^{+} \pi^{-}, K^{+} K^{-}$, and $p \bar{p}$, respectively. These efficiencies are only mildly dependent on the dihadron invariant mass and are very similar for the continuum background events. The continuum-subtracted dihadron invariant-mass plots are obtained by efficiency correcting each bin of the di-hadron invariant-mass plots for the $Y(1 \mathrm{~S})$ and $\sqrt{s}=10.56 \mathrm{GeV}$ data sets, scaling the latter plot by a factor of $0.404 \pm 0.002,{ }^{4}$ and subtracting it from the $Y(1 S)$ data set invariant-mass plot. Possible signals are determined by fitting each spectrum to spin-

\footnotetext{
${ }^{4}$ We obtain this factor, $f$, from the integrated luminosities of the $\mathrm{Y}(1 \mathrm{~S})$ and $\sqrt{s}=10.56 \mathrm{GeV}$ data sets, and the assumption that, to first order, the cross sections of the continuum processes in each run are proportional to $1 / s$. This factor is roughly equal to the factor obtained by using the average energy of each data set,

$$
f=0.404 \approx \frac{1.13 \mathrm{fb}^{-1}}{3.49 \mathrm{fb}^{-1}}\left(\frac{10.56 \mathrm{GeV}}{9.46 \mathrm{GeV}}\right)^{2} .
$$
}




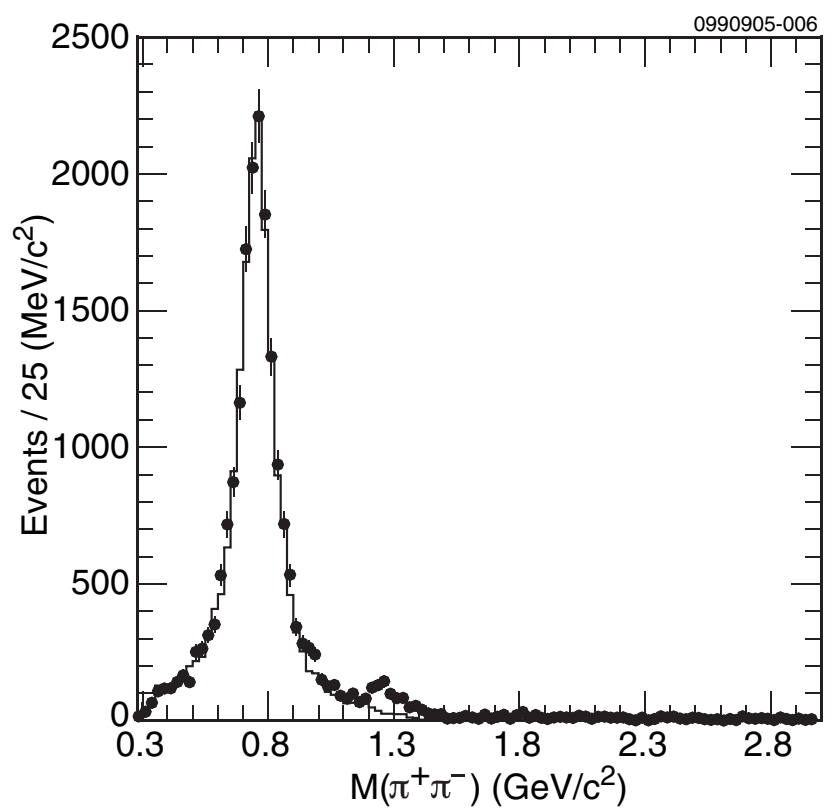

FIG. 1. Invariant mass of $\pi^{+} \pi^{-}$from $e^{+} e^{-} \rightarrow \gamma \pi^{+} \pi^{-}$for the scaled $\sqrt{s}=10.56 \mathrm{GeV}$ data set (solid line), and the $\mathrm{Y}(1 \mathrm{~S})$ data set (circles). The large number of events near $770 \mathrm{MeV} / \mathrm{c}^{2}$ is due to the abundant process $e^{+} e^{-} \rightarrow \gamma \rho$.

dependent relativistic Breit-Wigner functions. ${ }^{5}$ The spin value for each Breit-Wigner function is surmised by identifying each possible resonance in the invariant-mass plot based on its approximate mass and width. Later, we confirm these spin assignments for the significant resonances by inspecting the angular distributions of the $\mathrm{Y}(1 \mathrm{~S})$ decay products.

The $\pi^{+} \pi^{-}$invariant-mass plots for the $\mathrm{Y}(1 \mathrm{~S})$ and the scaled $\sqrt{s}=10.56 \mathrm{GeV}$ data sets are shown in Fig. 1. The fit to the continuum-subtracted $\pi^{+} \pi^{-}$spectrum, shown in Fig. 2, has a significant $f_{2}(1270)$ signal of $944 \pm 74$ events. It also has two less significant signal candidates: $340_{-130}^{+140}$ events in the $f_{0}(980)$ region, and $80 \pm 30$ events in the

${ }^{5}$ The spin-dependent relativistic Breit-Wigner parametrization used has the following probability distribution for a particular $h^{+} h^{-}$invariant-mass $x>x_{0}$,

where

$$
d P(x) \propto \frac{x x_{m} \Gamma(x)}{\left(x^{2}-x_{m}^{2}\right)^{2}+\left(x_{m} \Gamma(x)\right)^{2}} d x,
$$

$$
\Gamma(x)=\Gamma_{0}\left(\frac{x-x_{0}}{x_{m}-x_{0}}\right)^{2 S+1} \frac{2\left(x_{m}-x_{0}\right)^{2}}{\left(x-x_{0}\right)^{2}+\left(x_{m}-x_{0}\right)^{2}} .
$$

In the above expression, $x_{m}$ and $\Gamma_{0}$ represent, respectively, the most likely mass and width, and are allowed to float during the fit. The values of $x_{0}$ and $S$ are fixed during the fit to the invariantmass threshold for the particular mode and the spin of the resonance, respectively. The number of events for each fitted signal candidate is obtained by integrating this Breit-Wigner parametrization between threshold and $3 \mathrm{GeV} / \mathrm{c}^{2}$.

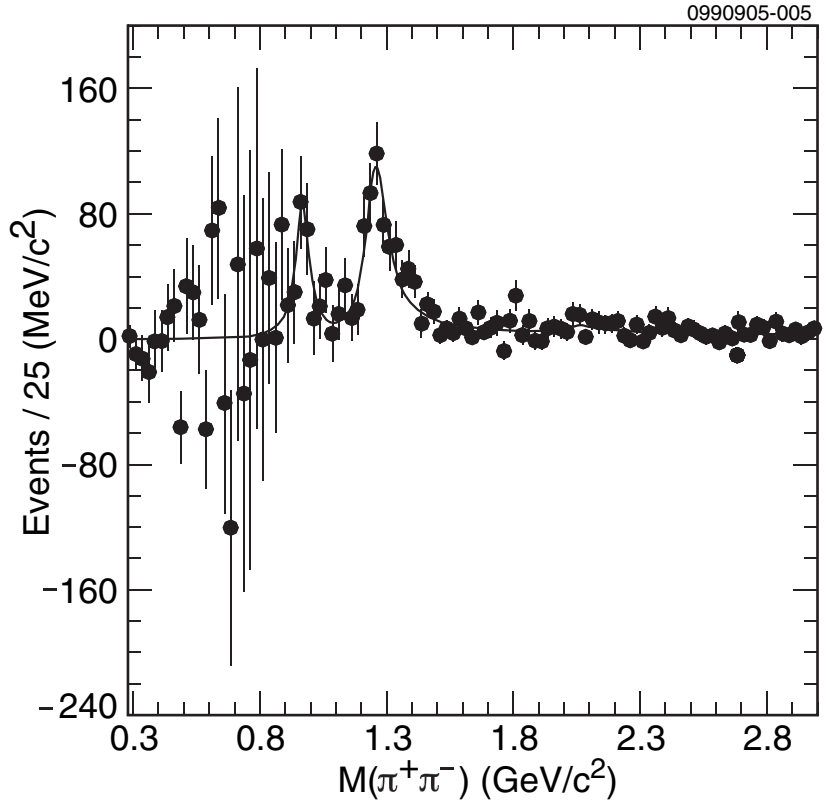

FIG. 2. Invariant mass of $\pi^{+} \pi^{-}$from $\Upsilon(1 S) \rightarrow \gamma \pi^{+} \pi^{-}$and the fit to the three spin-dependent relativistic Breit-Wigner functions described in the text.

$f_{4}(2050)$ region (see Fig. 3) whose significances are $4.3 \sigma$ and $2.6 \sigma$, respectively. Each significance is obtained by doing multiple chi-squared fits to the invariant-mass plot fixing the signal area to different values, assigning each of these multiple fits a probability proportional to $e^{-\chi^{2} / 2}$,

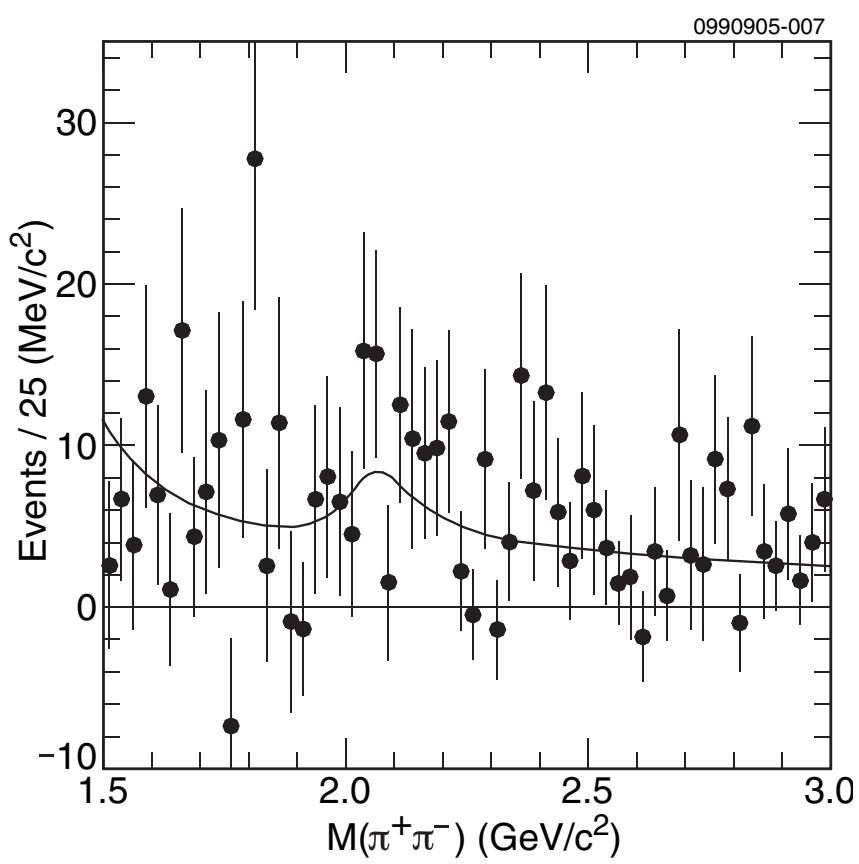

FIG. 3. Invariant mass of $\pi^{+} \pi^{-}$from $Y(1 \mathrm{~S}) \rightarrow \gamma \pi^{+} \pi^{-}$in the region $1.5-3.0 \mathrm{GeV} / \mathrm{c}^{2}$. This fit shows the small, nonsignificant, excess found in the $f_{4}(2050)$ region. 
normalizing the resulting probability distribution, and calculating the probability for negative or 0 signal.

The $K^{+} K^{-}$invariant-mass plots for the $\mathrm{Y}(1 \mathrm{~S})$ and the scaled $\sqrt{s}=10.56 \mathrm{GeV}$ data sets are shown in Fig. 4. The fit to the continuum-subtracted $K^{+} K^{-}$spectrum, shown in Fig. 5, has a significant signal of $312_{-61}^{+69}$ events identified as the $f_{2}^{\prime}(1525)$, and two nonsignificant signal candidates indicating possible $f_{2}(1270)$ and $f_{0}(1710)$ production with $109 \pm 36$ and $73 \pm 29$ events whose significances are $3.2 \sigma$ and $3.3 \sigma$, respectively. The excess of events in the $f_{2}(1270)$ region is consistent with that expected using the $\gamma \pi^{+} \pi^{-}$data and the known branching ratios for the $f_{2}(1270)$. We also note that there is a significant excess of $220 \pm 20$ events above $2.0 \mathrm{GeV} / \mathrm{c}^{2}$ in the $K^{+} K^{-}$ invariant-mass distribution which is not associated with any resonant structure.

The $p \bar{p}$ invariant-mass plots for the $\mathrm{Y}(1 \mathrm{~S})$ and the scaled $\sqrt{s}=10.56 \mathrm{GeV}$ data sets are shown in Fig. 6. No recognizable structure is seen in the continuum-subtracted $p \bar{p}$ spectrum, which is shown in Fig. 7. In particular, we do not see an enhancement near threshold, as might be expected from the BES $X(1860)$ results [57]. There is a nonsignificant excess of $85 \pm 18$ events in the $2-3 \mathrm{GeV} / \mathrm{c}^{2}$ invariantmass region.

To confirm the spins of our $f_{2}(1270) \rightarrow \pi^{+} \pi^{-}$and $f_{2}^{\prime}(1525) \rightarrow K^{+} K^{-}$signals, we examine the absolute value of the cosine of the polar angle of the photon with respect to the beam axis, $\left|\cos \theta_{\gamma}\right|$, and the absolute value of the

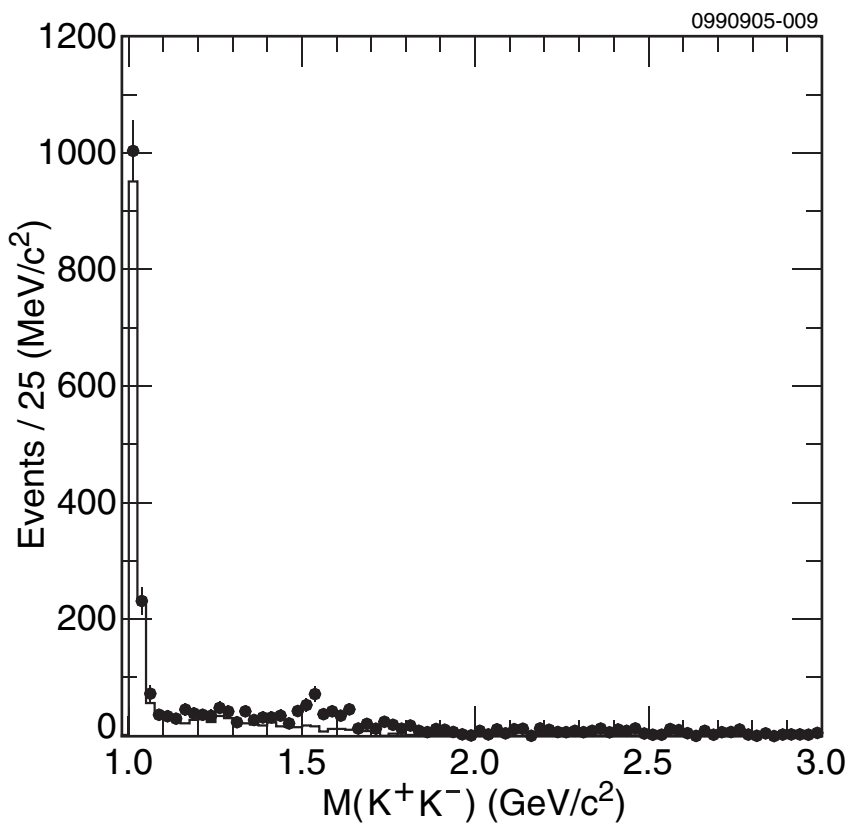

FIG. 4. Invariant mass of $K^{+} K^{-}$from $e^{+} e^{-} \rightarrow \gamma K^{+} K^{-}$for the scaled $\sqrt{s}=10.56 \mathrm{GeV}$ data set (solid line), and the $Y(1 \mathrm{~S})$ data set (circles). The large number of events near $1.050 \mathrm{GeV} / \mathrm{c}^{2}$ is due to the abundant process $e^{+} e^{-} \rightarrow \gamma \phi$.

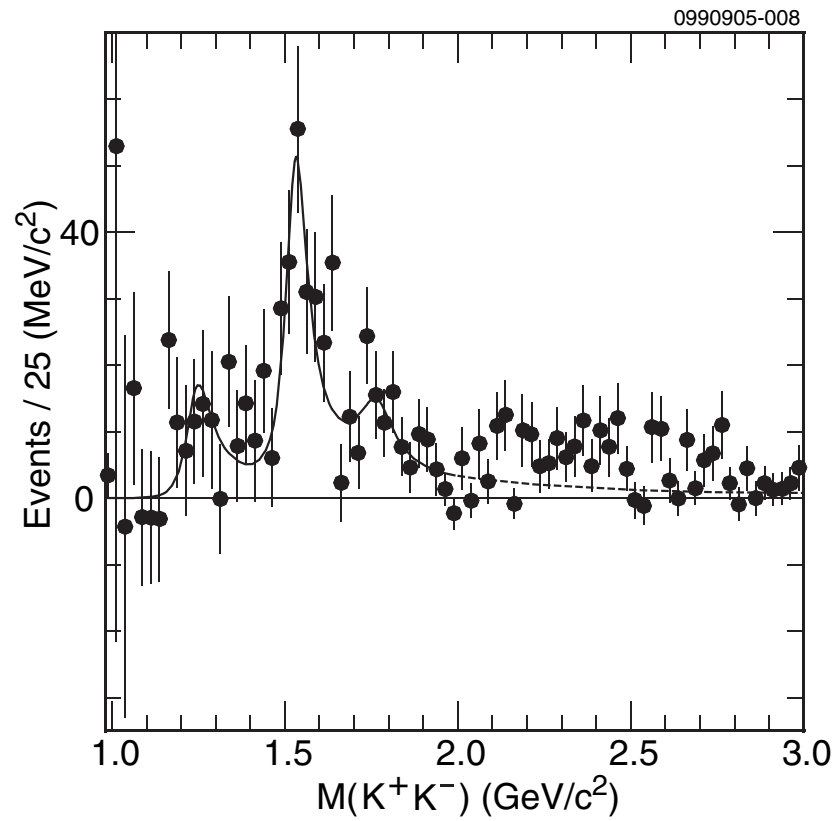

FIG. 5. Invariant mass of $K^{+} K^{-}$from $Y(1 S) \rightarrow \gamma K^{+} K^{-}$and the fit to the three spin-dependent relativistic Breit-Wigner functions described in the text. The dotted line shows the extrapolation of this fit to masses above $2 \mathrm{GeV} / \mathrm{c}^{2}$, which is the cutoff for the fit.

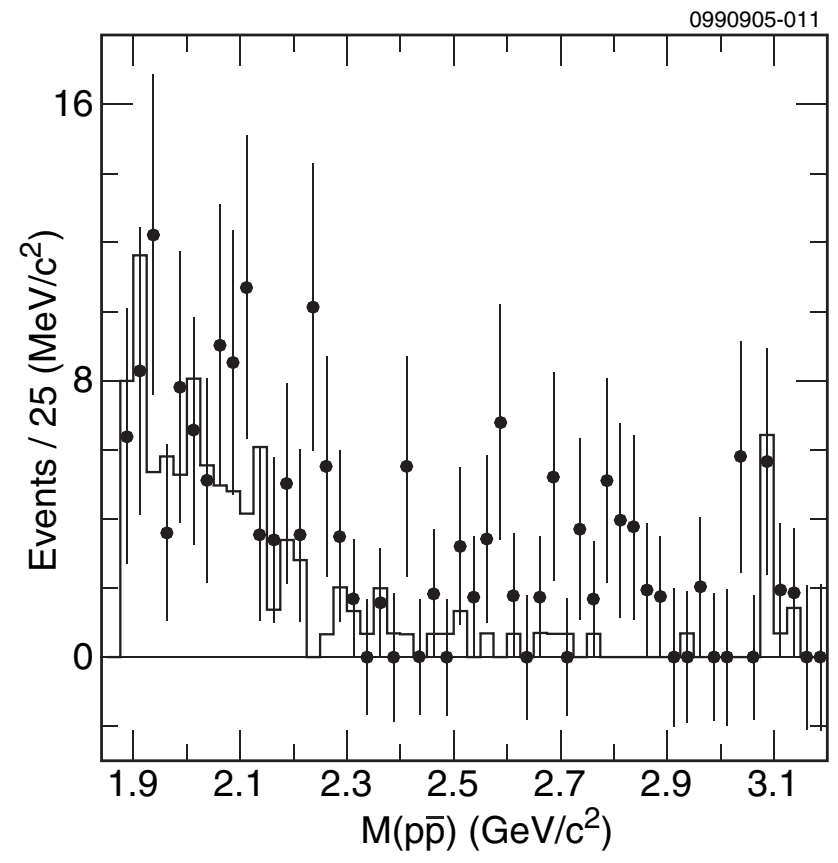

FIG. 6. Invariant mass of $p \bar{p}$ from $e^{+} e^{-} \rightarrow \gamma p \bar{p}$ for the scaled $\sqrt{s}=10.56 \mathrm{GeV}$ data set (solid line), and the $\mathrm{Y}(1 \mathrm{~S})$ data set (circles). The events near $3.1 \mathrm{GeV} / \mathrm{c}^{2}$ are due to the process $e^{+} e^{-} \rightarrow \gamma J / \psi$. 


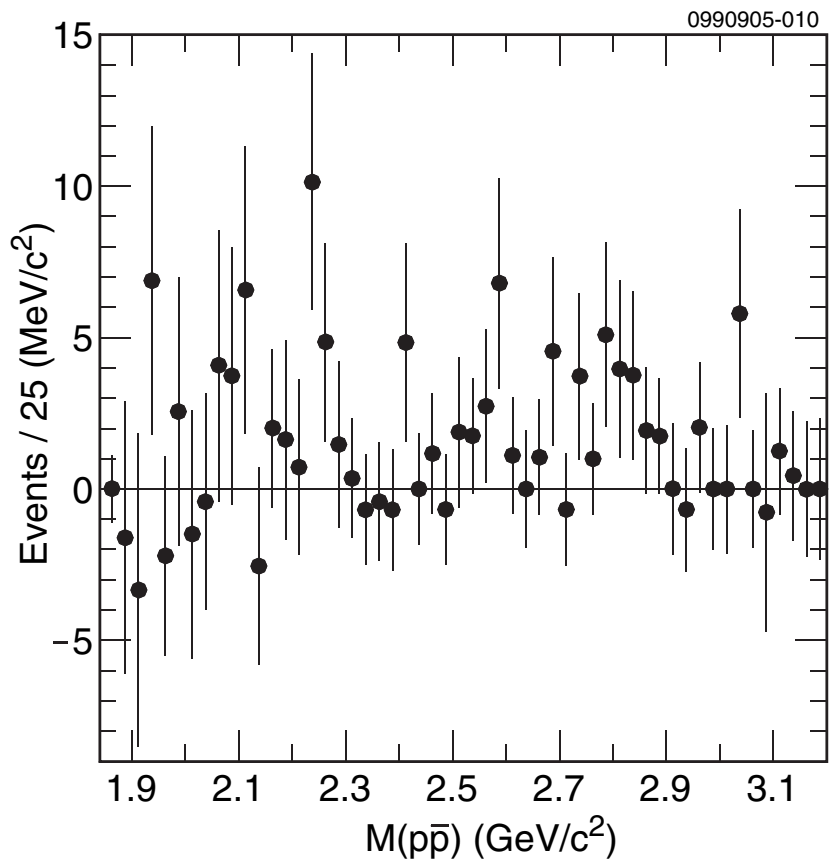

FIG. 7. Invariant mass of $p \bar{p}$ from $\Upsilon(1 \mathrm{~S}) \rightarrow \gamma p \bar{p}$.

cosine of the angle formed by the 3-momentum vector of one of the hadrons measured in the di-hadron rest frame with the photon's direction, $\left|\cos \theta_{h}\right|$. The event selection efficiency is slightly dependent on both angles, so to minimize systematic effects, the $\left|\cos \theta_{\gamma}\right|$ and $\left|\cos \theta_{h}\right|$ efficiency-corrected distributions are obtained by projecting the 2-dimensional bin-by-bin efficiency-corrected $\left(\left|\cos \theta_{\gamma}\right|,\left|\cos \theta_{h}\right|\right)$ distribution. We also subtract the background contributions from the tails of nearby resonances. The resulting angular distributions (shown in Figs. 8 and 9) are simultaneously fit to the helicity formalism prediction $[53,67,73]$ for different resonance spin hypotheses up to $J=4$. For the $f_{2}(1270)$ the different fit confidence levels are $8 \times 10^{-19}, 2 \times 10^{-19}, 0.05,8 \times 10^{-12}$, and $1 \times 10^{-12}$ for the hypotheses $J=0,1,2,3,4$, respectively. For the $f_{2}^{\prime}(1525)$ the different fit confidence levels are $2 \times 10^{-4}$, $2 \times 10^{-4}, 0.23,8 \times 10^{-3}$, and $2 \times 10^{-3}$ for the hypotheses $J=0,1,2,3,4$, respectively. These results confirm our identification of the resonances, as in both cases the angular distributions of the data strongly favor the $J=2$ hypothesis,

\footnotetext{
${ }^{6}$ Some authors use a probability distribution that also depends on a third angle, $\phi_{h}[50]$. However, extreme care must be taken when using this angle because it makes the probability distribution sensitive to the relative phases of the helicity amplitudes. Thus, two new free parameters need to be introduced in such a probability distribution, as was noted by [53] and correctly implemented by $[49,50]$. Otherwise, the measurement of the helicity amplitudes rests on the assumption that their relative phases are $0[46,48,74,75]$.
}

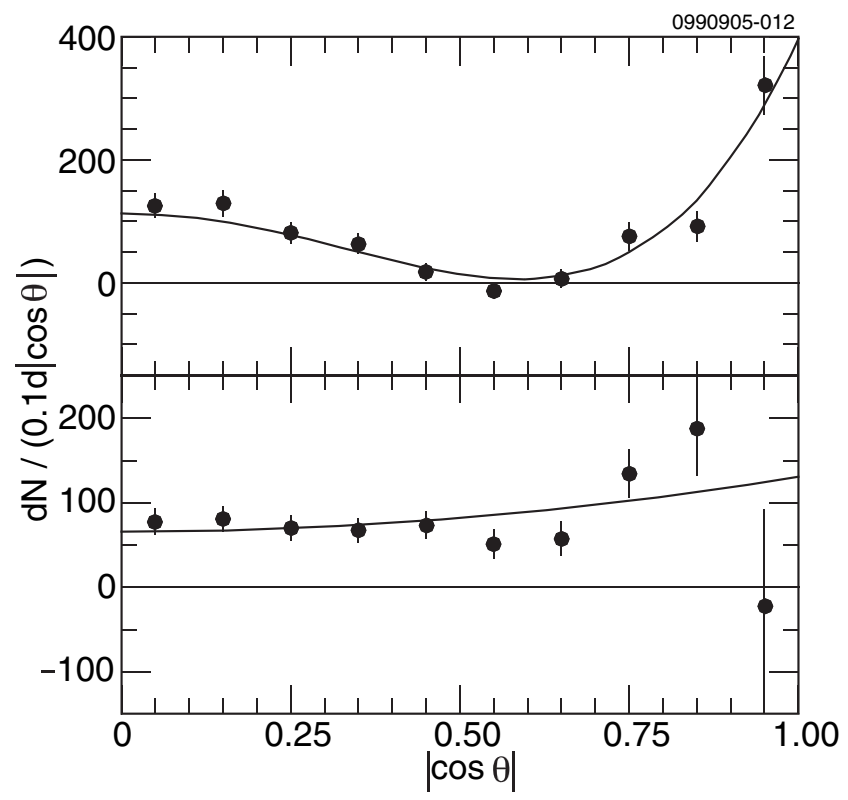

FIG. 8. Distributions of $\left|\cos \theta_{\pi}\right|$ (top) and $\left|\cos \theta_{\gamma}\right|$ (bottom) for the signal events in the $f_{2}(1270)$ invariant-mass region. The solid lines correspond to a simultaneous fit to the $J=2$ helicity formalism prediction [Eq. (3)].

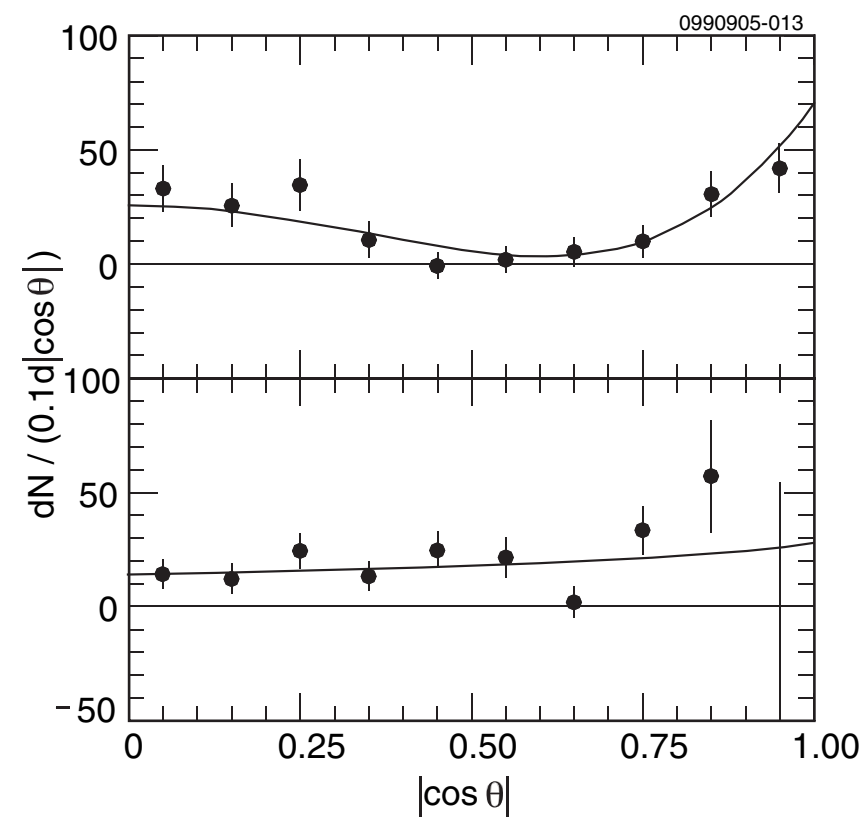

FIG. 9. Distributions of $\left|\cos \theta_{K}\right|$ (top) and $\left|\cos \theta_{\gamma}\right|$ (bottom) for the signal events in the $f_{2}^{\prime}(1525)$ invariant-mass region. The solid lines correspond to a simultaneous fit to the $J=2$ helicity formalism prediction [Eq. (3)]. 


$$
\begin{aligned}
\frac{d P_{\theta_{h}, \theta_{\gamma}}}{d \cos \theta_{h} d \cos \theta_{\gamma}}= & \left|a_{0}\right|^{2} \times \frac{5}{8}\left(3 \cos ^{2} \theta_{h}-1\right)^{2} \times \frac{3}{8}\left(1+\cos ^{2} \theta_{\gamma}\right) \\
& +\left|a_{1}\right|^{2} \times \frac{15}{16} \sin ^{2} 2 \theta_{h} \times \frac{3}{4} \sin ^{2} \theta_{\gamma}+\left|a_{2}\right|^{2} \\
& \times \frac{15}{16} \sin ^{4} \theta_{h} \times \frac{3}{8}\left(1+\cos ^{2} \theta_{\gamma}\right),
\end{aligned}
$$

where $a_{\lambda}, \lambda=0,1,2$, are the normalized helicity amplitudes, $\int d P_{\theta_{h}, \theta_{\gamma}}=\left|a_{0}\right|^{2}+\left|a_{1}\right|^{2}+\left|a_{2}\right|^{2}=1$. In other words, $\left|a_{\lambda}\right|^{2}$ is the probability of $X$ in $\mathrm{Y}(1 \mathrm{~S}) \rightarrow \gamma X$ to have helicity $\pm \lambda$. Because of the normalization condition, the $\left(\theta_{h}, \theta_{\gamma}\right)$ probability distribution can be described by two free parameters, traditionally chosen to be the helicity production ratios,

$$
x^{2}=\frac{\left|a_{1}\right|^{2}}{\left|a_{0}\right|^{2}} \quad \text { and } \quad y^{2}=\frac{\left|a_{2}\right|^{2}}{\left|a_{0}\right|^{2}} .
$$

To measure $x^{2}$ and $y^{2}$, we simultaneously fit the data to the individual $\theta_{h}$ and $\theta_{\gamma}$ distributions using ${ }^{7}$

$$
\begin{aligned}
\frac{d N_{\theta_{\gamma}}}{d \cos \theta_{\gamma}}= & N \int_{\theta_{h}} d P_{\theta_{h}, \theta_{\gamma}} \\
= & \frac{N}{1+x^{2}+y^{2}}\left[\frac{3}{8}\left(1+y^{2}\right)\left(1+\cos ^{2} \theta_{\gamma}\right)\right. \\
& \left.+\frac{3}{4} x^{2} \sin ^{2} \theta_{h}\right], \\
\frac{d N_{\theta_{h}}}{d \cos \theta_{h}}= & N \int_{\theta_{\gamma}} d P_{\theta_{h}, \theta_{\gamma}} \\
= & \frac{N}{1+x^{2}+y^{2}}\left[\frac{5}{8}\left(3 \cos ^{2} \theta_{h}-1\right)^{2}\right. \\
& \left.+\frac{15}{16} x^{2} \sin ^{2} 2 \theta_{h}+\frac{15}{16} y^{2} \sin ^{4} \theta_{h}\right],
\end{aligned}
$$

where $N$ corresponds to the number of events. Using the fits to the data (see Figs. 8 and 9) we measure the following helicity production ratios:

$$
\begin{aligned}
& x_{f_{2}(1270)}^{2}=0.00_{-0.00-0.00}^{+0.02+0.01}, \quad y_{f_{2}(1270)}^{2}=0.09_{-0.07-0.03}^{+0.08+0.04} \\
& x_{f_{2}^{\prime}(1525)}^{2}=0.00_{-0.00-0.00}^{+0.10+0.01}, \quad y_{f_{2}^{\prime}(1525)}^{2}=0.30_{-0.17-0.06}^{+0.22+0.07}
\end{aligned}
$$

where the first uncertainty is statistical and the second is systematic. The systematic uncertainty is quantified by

\footnotetext{
${ }^{7} \mathrm{We}$ choose to use a simultaneous fit to these two distributions instead of a two-dimensional fit using Eq. (2) because of our limited statistics.
}

studying how well input values are reproduced when analyzing Monte Carlo samples, the measured angular distribution of the photon and tracks from $e^{+} e^{-} \rightarrow \gamma \rho, \gamma \phi$ in the $\sqrt{s}=10.56$ data set, the effect of a one-sigma variation in the continuum scale factor, and possible interference with nearby resonances. We find that possible interference with nearby resonances in the $\left|\cos \theta_{h}\right|$ distribution dominates the systematic uncertainty. The helicity production ratio measurements indicate that both resonances are predominantly produced with helicity 0 . They are in agreement with the predictions of [2], and in good agreement with the twist-two-order predictions of [3]: no $\lambda=1$ production, and $\lambda=2$ production suppressed by a factor of $\left(m_{X} / m_{b}\right)^{2}$ with respect to $\lambda=0$ production, where $m_{X}$ is the mass of the tensor meson and $m_{b}$ is the mass of the $b$ quark.

We use the results from fitting the angular distributions to correct the Monte Carlo simulation efficiencies, which are calculated using flat distributions in the relevant angles, by a factor of $0.78 \pm 0.02$ for the $f_{2}(1270), 0.90 \pm 0.01$ for the $f_{2}^{\prime}(1525)$, and $0.88_{-0.01}^{+0.03}$ for the significant excess in the $2-3 \mathrm{GeV} / \mathrm{c}^{2}$ region of the di-kaon invariant mass. The large correction in the pion mode is due to the necessarily stronger muon suppression requirement. The measured branching ratios of the significant resonances are

$$
\begin{aligned}
& \mathcal{B}\left(\Upsilon(1 S) \rightarrow \gamma f_{2}(1270)\right)=(10.2 \pm 0.8 \pm 0.7) \times 10^{-5} \\
& \mathcal{B}\left(\Upsilon(1 S) \rightarrow \gamma f_{2}^{\prime}(1525)\right)=\left(3.7_{-0.7}^{+0.9} \pm 0.8\right) \times 10^{-5}
\end{aligned}
$$

and the measured branching ratio of the excess events in $\Upsilon(1 \mathrm{~S}) \rightarrow \gamma K^{+} K^{-}$with di-kaon invariant mass between $2-3 \mathrm{GeV} / \mathrm{c}^{2}$ is

$$
\mathcal{B}\left(\Upsilon(1 \mathrm{~S}) \rightarrow \gamma K^{+} K^{-}\right)=(1.14 \pm 0.08 \pm 0.10) \times 10^{-5}
$$

where the first uncertainty is statistical and the second is systematic. The sources of systematic uncertainty are $1 \%$ from the number of $\mathrm{Y}(1 \mathrm{~S})$ decays, $2 \%$ from the Monte Carlo simulation of the track reconstruction, 3\% (8\%) from the Monte Carlo efficiency modeling of the event requirements in the pion (kaon) mode, and $1 \%$ to $3 \%$ from the uncertainty in the angular distribution measurements. We also assign a $15 \%$ systematic uncertainty to the $f_{2}^{\prime}(1525)$ branching fraction from possible interference between the $f_{2}(1270)$ and $f_{2}^{\prime}(1525)$ resonances, and less than a $1 \%$ systematic uncertainty from high-momentum neutral pions faking photons in the decay $\mathrm{Y}(1 \mathrm{~S}) \rightarrow \rho \pi$, based on the upper limit in [76]. Finally, we include the uncertainties in the $f_{2}(1270)$ and $f_{2}^{\prime}(1525)$ hadronic branching ratios [45] in the systematic uncertainty. For our less significant signal candidates, the branching fraction central values, along with their significances and $90 \%$ confidence level upper limits, are shown in Table I. 
TABLE I. Branching fraction central value (BF), its statistical significance, and its $90 \%$ confidence level upper limit (UL), for each signal candidate with a significance $<5 \sigma$. In the branching fraction central value, the first uncertainty is statistical and the second is systematic. The first three table entries are product branching fractions.

\begin{tabular}{lccc}
\hline \hline Channel & BF $\left(10^{-5}\right)$ & Significance & UL $\left(10^{-5}\right)$ \\
\hline$\Upsilon(1 \mathrm{~S}) \rightarrow \gamma f_{0}(980) \rightarrow \pi^{+} \pi^{-}$ & $1.8_{-0.7}^{+0.8} \pm 0.1$ & $4.3 \sigma$ & $<3$ \\
$\Upsilon(1 \mathrm{~S}) \rightarrow \gamma f_{4}(2050) \rightarrow \pi^{+} \pi^{-}$ & $0.37 \pm 0.14 \pm 0.03$ & $2.6 \sigma$ & $<0.6$ \\
$\Upsilon(1 \mathrm{~S}) \rightarrow \gamma f_{0}(1710) \rightarrow K^{+} K^{-}$ & $0.38 \pm 0.16 \pm 0.04$ & $3.2 \sigma$ & $<0.7$ \\
$Y(1 \mathrm{~S}) \rightarrow \gamma p \bar{p}, 2 \mathrm{GeV} / \mathrm{c}^{2}<m_{p \bar{p}}<3 \mathrm{GeV} / \mathrm{c}^{2}$ & $0.41 \pm 0.08 \pm 0.10$ & $4.8 \sigma$ & $<0.6$ \\
\hline \hline
\end{tabular}

\section{DETERMINATION OF UPPER LIMITS FOR $f_{J}(222)$ AND $X(1860)$ PRODUCTION AND DECAY}

To measure upper limits of the product branching ratio for the decays $Y(1 S) \rightarrow \gamma f_{J}(2220)$ with $f_{J}(2220) \rightarrow$ $h^{+} h^{-}$, we fit the $h^{+} h^{-}$invariant-mass plots, shown in Fig. 10, using a Breit-Wigner function with a peak mass and width fixed at $2.234 \mathrm{GeV} / \mathrm{c}^{2}$ and $0.017 \mathrm{GeV} / \mathrm{c}^{2}$, respectively. These are the values from the possible $f_{J}(2220)$ signal reported by the BES experiment [54], which is considered a candidate for a glueball. To model the general excess of events between 2.0 and $2.5 \mathrm{GeV} / \mathrm{c}^{2}$ we also use a flat background function in the fit. Although the highest bin in the $p \bar{p}$ plot is indeed in the region of the $f_{J}(2220)$, the excess (12 \pm 5 events) is not significant, and there are no

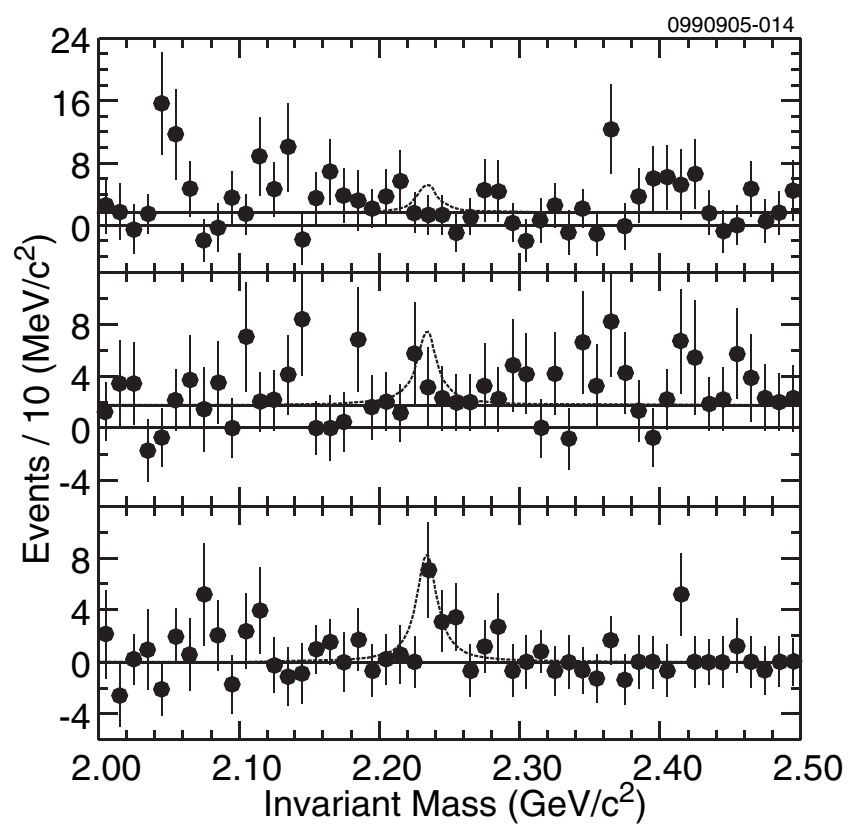

FIG. 10. Invariant mass of $\pi^{+} \pi^{-}$(top), $K^{+} K^{-}$(middle), and $p \bar{p}$ (bottom) from $Y(1 \mathrm{~S}) \rightarrow \gamma h^{+} h^{-}$in the range 2.0 to $2.5 \mathrm{GeV} / \mathrm{c}^{2}$. Also shown are the results of fits to a $f_{J}(2220)$ Breit-Wigner function (dashed lines) and a flat background (solid lines). The $f_{J}(2220)$ signal line shown corresponds to the $90 \%$ confidence level maximum yield obtained from the fit. The flat background function is also used to measure the possible excesses of events in the invariant-mass region 2 to $3 \mathrm{GeV} / \mathrm{c}^{2}$. significant signals anywhere in these three plots. To find upper limits for $f_{J}(2220) \rightarrow h^{+} h^{-}$decays, we fix the area of the Breit-Wigner function to different values, minimize the chi squared from the fit, and give that area a probability proportional to $e^{-\chi^{2} / 2}$. These probability distributions are then used to obtain the following $90 \%$ confidence level upper limits on the product branching ratio for $f_{J}(2220)$ production and decay to each mode:

$$
\begin{aligned}
\mathcal{B}(Y(1 S) & \left.\rightarrow \gamma f_{J}(2200)\right) \times \mathcal{B}\left(f_{J}(2200)\right. \\
& \left.\rightarrow \pi^{+} \pi^{-}\right)<8 \times 10^{-7} \\
\mathcal{B}(Y(1 S) & \left.\rightarrow \gamma f_{J}(2200)\right) \times \mathcal{B}\left(f_{J}(2200)\right. \\
& \left.\rightarrow K^{+} K^{-}\right)<6 \times 10^{-7}, \\
\mathcal{B}(\mathrm{Y}(1 \mathrm{~S}) & \left.\rightarrow \gamma f_{J}(2200)\right) \times \mathcal{B}\left(f_{J}(2200)\right. \\
& \rightarrow p \bar{p})<11 \times 10^{-7} .
\end{aligned}
$$

The systematic uncertainties on the branching ratios were added in quadrature with the statistical errors in forming the above limits. Using the $X(1860)$ parameters measured in [57], and proceeding in a similar manner as described above, we obtain

$$
\begin{aligned}
\mathcal{B}(\mathrm{Y}(1 \mathrm{~S}) & \rightarrow \gamma X(1860)) \times \mathcal{B}(X(1860) \\
& \rightarrow p \bar{p})<5 \times 10^{-7},
\end{aligned}
$$

at the $90 \%$ confidence level.

\section{SUMMARY AND CONCLUSION}

We have confirmed CLEO's previous observation of the $f_{2}(1270)$ in radiative $\mathrm{Y}(1 \mathrm{~S})$ decays and made a new observation of the $f_{2}^{\prime}(1525)$, obtaining factors of $0.07 \pm 0.01$ and $0.08_{-0.03}^{+0.04}$ for the ratio of the $\mathrm{Y}(1 \mathrm{~S})$ branching fraction with respect to the one measured in $J / \psi$ radiative decays, respectively. These values are larger than, but the same order of magnitude as, the ratio of 0.04 expected from naive scaling arguments. The observed $f_{2}(1270)$ production is in agreement with the prediction in [3] and somewhat lower than the prediction in [2]. In both of the measured modes we can confirm by fits to the angular distributions of the photon and charged particles that the 
two daughter hadrons are indeed produced by a spin-2 parent. We find that this parent is produced mostly with helicity 0 , in good agreement with the predictions in $[2,3]$. No structure is seen in the $p \bar{p}$ invariant-mass distribution. In particular, we do not observe a near-threshold enhancement as in [57]. Finally, stringent limits have been put on the production of the glueball candidate $f_{J}(2220)$ in radiative $\mathrm{Y}(1 \mathrm{~S})$ decays. Glueball production is expected to be enhanced in $Y(1 S)$ radiative decays $[22,77,78]$, but we find that, within our experimental sensitivity, known tensor meson states, believed to be composed only of quarks, dominate the di-gluon spectrum.

\section{ACKNOWLEDGMENTS}

We gratefully acknowledge the effort of the CESR staff in providing us with excellent luminosity and running conditions. This work was supported by the National Science Foundation and the U.S. Department of Energy.
[1] G. T. Bodwin, E. Braaten, and G. P. Lepage, Phys. Rev. D 51, 1125 (1995).

[2] S. Fleming, C. Lee, and A. K. Leibovich, Phys. Rev. D 71, 074002 (2005).

[3] J. P. Ma, Nucl. Phys. B605, 625 (2001).

[4] V. N. Baier and A. G. Grozin, Sov. J. Nucl. Phys. 35, 899 (1982).

[5] V. N. Baier and A. G. Grozin, Z. Phys. C 29, 161 (1985).

[6] T. Barnes, Z. Phys. C 10, 275 (1981).

[7] J. M. Cornwall and A. Soni, Phys. Lett. B 120, 431 (1983).

[8] W. S. Hou and G. G. Wong, Phys. Rev. D 67, 034003 (2003).

[9] C. J. Morningstar and M. J. Peardon, Phys. Rev. D 60, 034509 (1999).

[10] A. Vaccarino and D. Weingarten, Phys. Rev. D 60, 114501 (1999).

[11] C. Liu, Commun. Theor. Phys. 35, 288 (2001); Chin. Phys. Lett. 18, 187 (2001).

[12] D. Q. Liu, J. M. Wu, and Y. Chen, High Energy Phys. Nucl. Phys. 26, 222 (2002).

[13] P. G. O. Freund and Y. Nambu, Phys. Rev. Lett. 34, 1645 (1975).

[14] R. L. Jaffe and K. Johnson, Phys. Lett. B 60, 201 (1976).

[15] T. Barnes, F.E. Close, and S. Monaghan, Nucl. Phys. B198, 380 (1982).

[16] C. E. Carlson, T. H. Hansson, and C. Peterson, Phys. Rev. D 27, 1556 (1983).

[17] N. Isgur and J. Paton, Phys. Rev. D 31, 2910 (1985).

[18] S. Narison, Z. Phys. C 26, 209 (1984); Nucl. Phys. B509, 312 (1998); Nucl. Phys. B, Proc. Suppl. 96, 244 (2001).

[19] M. H. Thomas, M. Lust, and H. J. Mang, J. Phys. G 18, 1125 (1992).

[20] J. Y. Cui, J. M. Wu, and H. Y. Jin, Phys. Lett. B 424, 381 (1998).

[21] S. J. Brodsky, A. S. Goldhaber, and J. Lee, Phys. Rev. Lett. 91, 112001 (2003).

[22] X. G. He, H. Y. Jin, and J. P. Ma, Phys. Rev. D 66, 074015 (2002).

[23] M. Melis, F. Murgia, and J. Parisi, Phys. Rev. D 70, 034021 (2004).

[24] J. C. Su and J. X. Chen, Phys. Rev. D 69, 076002 (2004).

[25] V. V. Anisovich et al. (Crystal Barrel Collaboration), Phys. Lett. B 323, 233 (1994).

[26] V. V. Anisovich, D. V. Bugg, A. V. Sarantsev, and B. S.
Zou, Phys. Rev. D 50, 1972 (1994).

[27] C. Amsler et al. (Crystal Barrel Collaboration), Phys. Lett. B 355, 425 (1995).

[28] C. A. Meyer, AIP Conf. Proc. 698, 554 (2004).

[29] K. K. Seth, Nucl. Phys. B, Proc. Suppl. 96, 205 (2001).

[30] B. S. Zou, Nucl. Phys. A655, 41 (1999).

[31] A. Etkin et al., Phys. Lett. B 201, 568 (1988); Phys. Lett. B 165, 217 (1985).

[32] C. Amsler and F. E. Close, Phys. Lett. B 353, 385 (1995).

[33] D. Weingarten, Nucl. Phys. B, Proc. Suppl. 53, 232 (1997).

[34] D. V. Bugg, M. J. Peardon, and B. S. Zou, Phys. Lett. B 486, 49 (2000).

[35] J. Sexton, A. Vaccarino, and D. Weingarten, Phys. Rev. Lett. 75, 4563 (1995).

[36] V. V. Anisovich, Phys. Lett. B 364, 195 (1995).

[37] F. Giacosa, T. Gutsche, and A. Faessler, Phys. Rev. C 71, 025202 (2005).

[38] M. Chanowitz, Phys. Rev. Lett. 95, 172001 (2005).

[39] A. H. Fariborz, Int. J. Mod. Phys. A 19, 5417 (2004).

[40] A. V. Anisovich, V. V. Anisovich, Y. D. Prokoshkin, and A. V. Sarantsev, Z. Phys. A 357, 123 (1997); Nucl. Phys. Proc. Suppl. A 56, 270 (1997).

[41] D. Weingarten, hep-ph/9607212.

[42] V. V. Anisovich, M. A. Matveev, J. Nyiri, and A. V. Sarantsev, Int. J. Mod. Phys. A 20, 6327 (2005); V. V. Anisovich and A. V. Sarantsev, Pis'ma Zh. Eksp. Teor. Fiz. 81, 531 (2005) [JETP Lett. 81, 417 (2005)].

[43] Y.D. Prokoshkin et al. (GAMS Collaboration), Phys. Dokl. 40, 495 (1995).

[44] N. Brambilla et al., hep-ph/0412158.

[45] S. Eidelman et al. (Particle Data Group Collaboration), Phys. Lett. B 592, 1 (2004).

[46] G. Alexander et al. (Pluto Collaboration), Phys. Lett. B 76, 652 (1978)

[47] D. L. Scharre, in Proceedings of the 10th International Symposium on Lepton and Photon Interactions at High Energy, Bonn, 1981.

[48] C. Edwards et al. (Crystal Ball Collaboration), Phys. Rev. D 25, 3065 (1982)

[49] J. E. Augustin et al. (DM2 Collaboration), Z. Phys. C 36, 369 (1987).

[50] R. M. Baltrusaitis et al. (Mark III Collaboration), Phys. Rev. D 35, 2077 (1987) 
[51] J.Z. Bai et al. (BES Collaboration), Phys. Rev. D 68, 052003 (2003).

[52] M. Krammer, Phys. Lett. B 74, 361 (1978).

[53] J. G. Korner, J. H. Kuhn, M. Krammer, and H. Schneider, Nucl. Phys. B229, 115 (1983).

[54] J.Z. Bai et al. (BES Collaboration), Phys. Rev. Lett. 76, 3502 (1996).

[55] R. M. Baltrusaitis et al. (MARK-III Collaboration), Phys. Rev. Lett. 56, 107 (1986).

[56] J. E. Augustin et al. (DM2 Collaboration), Phys. Rev. Lett. 60, 2238 (1988).

[57] J.Z. Bai et al. (BES Collaboration), Phys. Rev. Lett. 91, 022001 (2003).

[58] A. Sibirtsev, J. Haidenbauer, S. Krewald, U. G. Meissner, and A. W. Thomas, Phys. Rev. D 71, 054010 (2005).

[59] B. Kerbikov, A. Stavinsky, and V. Fedotov, Phys. Rev. C 69, 055205 (2004).

[60] D. V. Bugg, Phys. Lett. B 598, 8 (2004).

[61] C. S. Gao and S. L. Zhu, Commun. Theor. Phys. 42, 844 (2004).

[62] B.S. Zou and H.C. Chiang, Phys. Rev. D 69, 034004 (2004).

[63] B. Loiseau and S. Wycech, Phys. Rev. C 72, 011001 (2005).

[64] G. J. Ding and M. L. Yan, Phys. Rev. C 72, 015208 (2005).

[65] A. Anastassov et al. (CLEO Collaboration), Phys. Rev. Lett. 82, 286 (1999).

[66] G. Masek et al. (CLEO Collaboration), Phys. Rev. D 65, 072002 (2002).
[67] L. Breva-Newell, Ph.D. thesis, University of Florida (hepex/0412075).

[68] Y. Kubota et al., Nucl. Instrum. Methods Phys. Res., Sect. A 320, 66 (1992); G. Viehhauser et al., Nucl. Instrum. Methods Phys. Res., Sect. A 462, 146 (2001); D. Peterson et al., Nucl. Instrum. Methods Phys. Res., Sect. A 478, 142 (2002); A. Warburton et al., Nucl. Instrum. Methods Phys. Res., Sect. A 488, 451 (2002); M. Artuso et al., Nucl. Instrum. Methods Phys. Res., Sect. A 502, 91 (2003); 554, 147 (2005).

[69] R. A. Briere et al. (CLEO Collaboration), Phys. Rev. D 70, 072001 (2004).

[70] QQ-The CLEO Event Generator, http://www.Ins.cornell. edu/public/CLEO/soft/QQ (unpublished).

[71] R. Brun et al., GEANT 3.21, CERN Program Library Long Writeup W5013, 1993 (unpublished).

[72] M. A. Selen, R. M. Hans, and M. J. Haney, IEEE Trans. Nucl. Sci. 48, 562 (2001).

[73] J.D. Richman, California Institute of Technology Report No. CALT-68-1148, 1984 (unpublished).

[74] P. K. Kabir and A. J. G. Hey, Phys. Rev. D 13, 3161 (1976).

[75] M. Ablikim et al. (BES Collaboration), Phys. Rev. D 70, 092004 (2004).

[76] S. A. Dytman et al. (CLEO Collaboration), hep-ex/ 0307035.

[77] S. Godfrey and J. Napolitano, Rev. Mod. Phys. 71, 1411 (1999).

[78] F. E. Close, G. R. Farrar, and Z. Li, Phys. Rev. D 55, 5749 (1997). 